\title{
Rosette-forming Glioneuronal Tumors in the Posterior Third Ventricle
}

\author{
Ibrahim Alnaami, Keith Aronyk, Jian-Qiang Lu, Edward S. Johnson, Cian O'Kelly
}

Can J Neurol Sci. 2013; 40: 885-888

Rosette-forming glioneuronal tumor (RGNT) is a rare brain tumor found almost exclusively within the fourth ventricle. These grade I tumors were first included in the World Health Organization (WHO) Classification for the central nervous system in $2007^{1}$. Since then, approximately 49 cases have been published $^{1,2}$.

There are no reported cases of RGNT within the third ventricle, although this tumor has also been described in the pineal region, the tectum and within the aqueduct ${ }^{2}$. Supratentorial localization is quite rare, with only three published: one was found in the septum pellucidum ${ }^{3}$, another within the optic nerve in neurofibromatosis type 1 (NF1) patient ${ }^{1}$ and one in a patient with multiple RGNT in the lateral ventricle ${ }^{2}$. The authors report the first case series in the literature of RGNT occurring within the posterior third ventricle.

\section{Case Series- Case 1}

History and Physical Examination. A 57-year-old male presented with complaints of progressive headaches over a few weeks. He also experienced vomiting and gait instability. There were no other neurological symptoms. On examination, his cranial nerves were all intact with no papilloedma. The remainder of the exam showed normal power, reflexes, coordination, and sensation in both upper and lower extremities.

Imaging. An initial non-contrast computed tomography (CT) scan of the head demonstrated obstructive hydrocephalus. Subsequent magnetic resonance imaging (MRI) of the brain (Figure 1), confirmed hydrocephalus but also demonstrated a lesion within the posterior aspect of the third ventricle. The lesion was isointense on T1 weighted sequences and hyperintense on $\mathrm{T} 2$ weighted sequences with a heterogeneous enhancement pattern following administration of gadolinium.

Operation. Given the progressive nature of the patient's symptoms, a decision was made to proceed with endoscopic third ventriculostomy (ETV) and biopsy of the lesion. The procedure was done using a flexible endoscope (Storz). By flexing the endoscope underneath the massa intermedia of the thalami, the tumor was visualized in the aqueductal recess, attached to the right thalamus (Figure 2). Three small biopsy samples were obtained. The endoscope was then directed

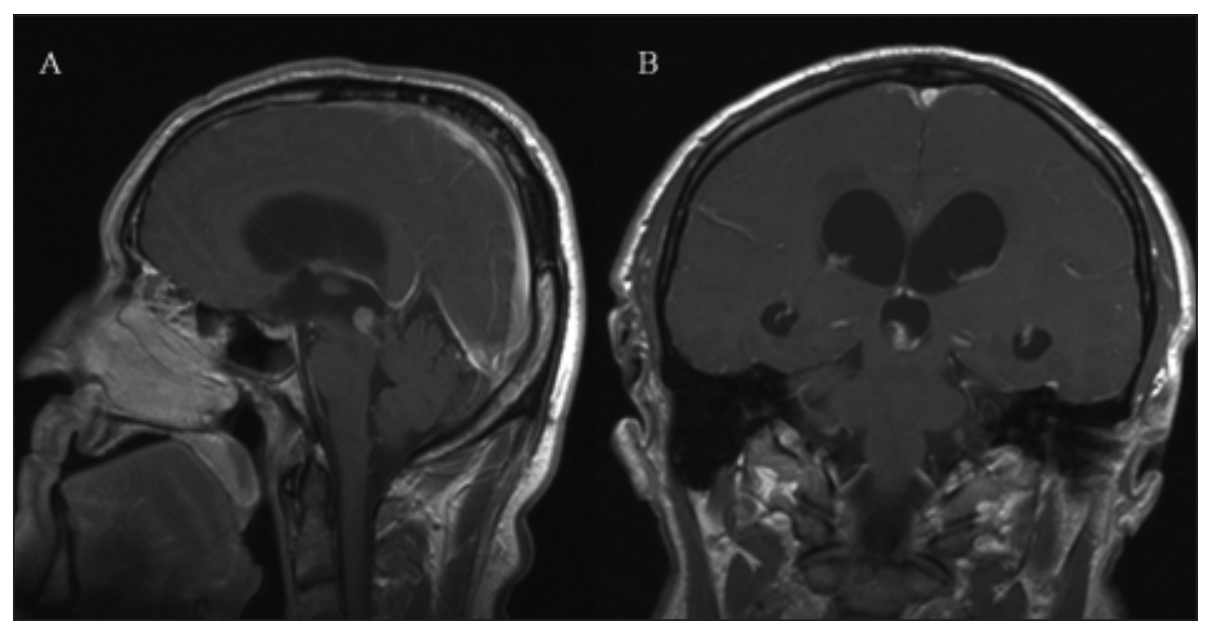

Figure 1: $A$ and B, pre-operative enhanced sagittal and coronal MRI, case 1, show an enhancing posterior third ventricular lesion with tri-ventricular hydrocephalus.

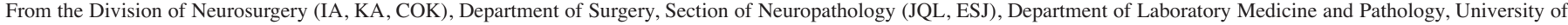
Alberta, Edmonton, Canada; Division of Neurosurgery (IA), Department of Surgery, King Khalid University, Abha, Saudi Arabia.

Received March 7, 2013. Final Revisions SubmitTed May 30, 2013.

Correspondence to: Ibrahim Alnaami, Neurosurgery Division, 2D2.01 Mackenzie Health Sciences Centre, University of Alberta, 8440 112 Street, Edmonton, Alberta, T6G 2B7,

Canada.Email: ialnaami@ualberta.ca.
} 


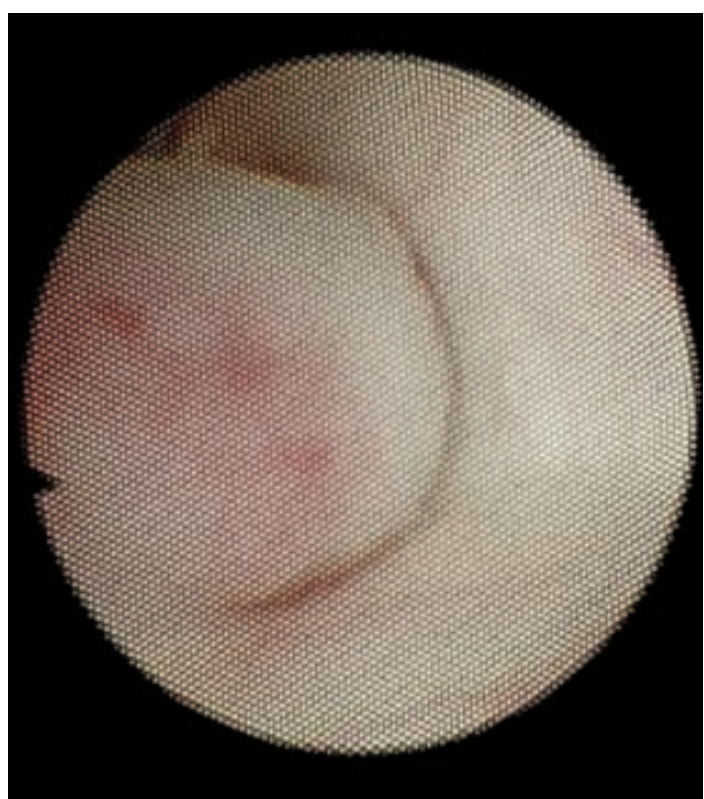

Figure 2: Endoscopic view of the posterior third ventricle with the tumor pedicle, case 1.

towards the floor of the third ventricle to complete the ventriculostomy.

Post-operative course. An external ventricular drain was left in place for 24 hours and then removed. The patient was discharged home in good condition on post-operative day two. He has remained free of symptoms in subsequent follow-up out to six months. A follow-up MRI of the brain demonstrated a functioning third ventriculostomy. The tumor itself remained stable in size and interestingly no longer enhances following gadolinium administration. (Figure 3)

\section{Case 2}

History and Physical Examination. A 28-year-old previously healthy male presented with headaches over the previous six weeks. The headache was worse with cough. He developed diplopia three days prior to his presentation. Examination revealed mild bilateral lateral gaze palsy. The remainder of the neurologic exam was otherwise normal.

Imaging. CT head and MRI showed enlargement of the third and lateral ventricles due to an expansile mass in the posterior third ventricle extending into the aqueduct. However, the possibility of this lesion arising from the aqueduct and extending into the posterior third ventricle was feasible. The lesion was hypointense on T1weighted sequence and hyperintense on T2 weighted sequence. Nodular enhancement in the centre of the lesion was seen following administration of gadolinium (Figure $4 \mathrm{~A}$, and B).

Operation. Endoscopic third ventriculoscopy (ETV) and biopsy of the lesion was undertaken. Storz flexible scope was utilized for the procedure. The scope was flexed to visualize the tumor within the aqueductal recess. Three small biopsy samples were obtained with no bleeding. Endoscopic third ventriculoscopy was performed following the biopsy. An external ventricular drain was not required.
Post-operative course. The patient was admitted to neurosurgery step-down unit for 24 hours for monitoring. A CT head on post-operative day one revealed diminished size of the ventricles. The patient was discharged home on post-operative day two.

Pathology Findings. Pathology examination of the small biopsy fragments from Cases 1 and 2 revealed similar findings of a glioneuronal tumor with mixed neurocytic and piloid astrocytic components. The neurocytic component is represented by fields of small polygonal neurocytic cells organized either in circlets around wispy eosinophilic neuropil cores to describe neurocytic rosette formations, or in collars around a central capillary to form perivascular pseudorosettes (Figures 5A and $5 \mathrm{~B}$; Figures $6 \mathrm{~A}$ and $6 \mathrm{~B})$. A characteristic finding is the marked expression of synaptophysin immunoreactivity within the neuropil cores of these neurocytic rosettes and, less consistently,

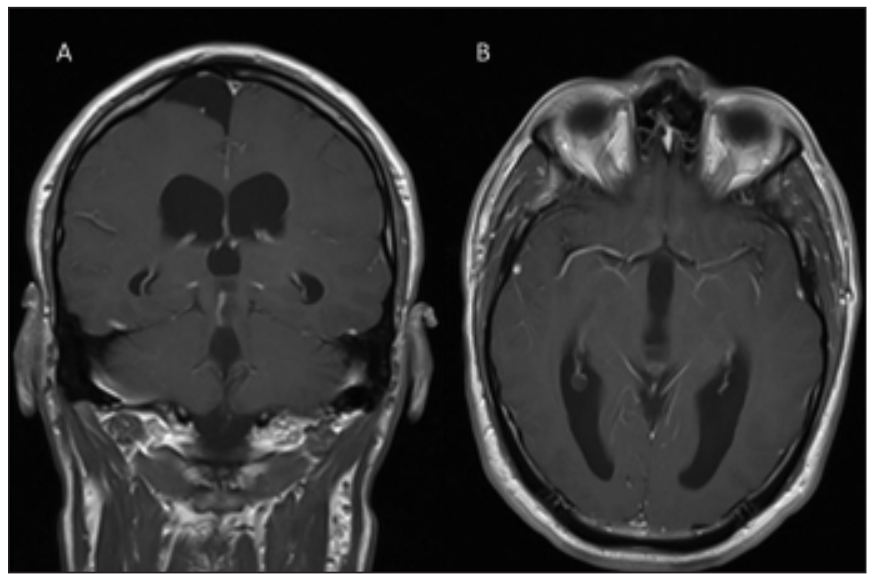

Figure 4: $A$ and B, pre-operative enhanced sagittal and coronal MRI of case 2 show a centrally enhancing posterior third ventricular lesion with tri-ventricular hydrocephalus. 


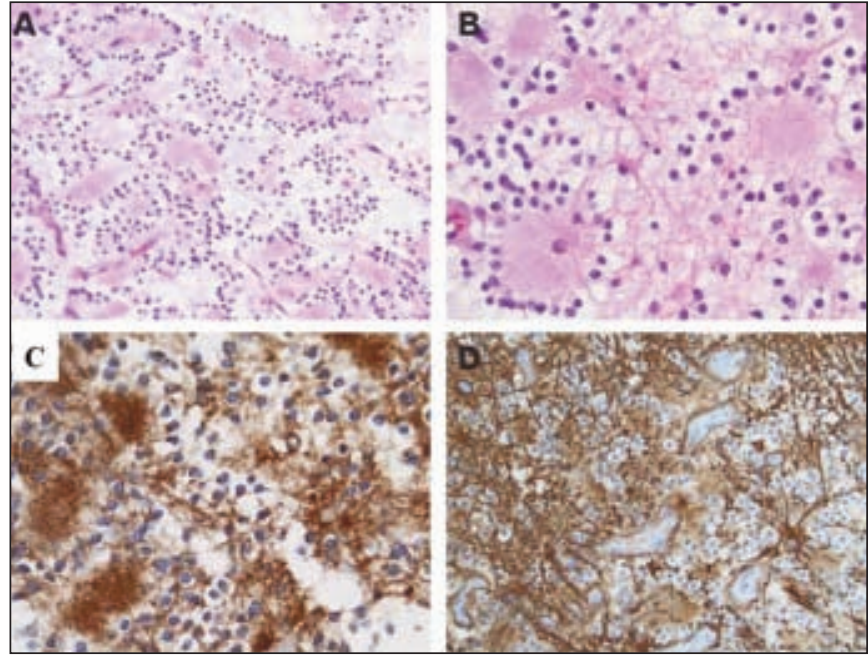

Figure 5: Histopathology of Rosette-forming glioneuronal tumor, case 1. $A$, well-differentiated cells forming neurocytic rosettes and perivascular pseudorossettes (hematoxylin and eosin, x 200). B, glial component containing microcytes, oligodendroglia-like cells and larger cells with cytoplasmic processes (hematoxylin and eosin, $x$ 400). C, Synaptophysin immunoreactivity in the centers of neurocytic rosettes, neuropils, and neurocytic cells (x 400). D, GFAP immunoreactivity in the glial component (x 200).

the perivascular pseudorosettes (Figure 5C and Figure 6C). The piloid glial component consists of mildly hypercellular sheets of spindled cells with intervening columns and clusters of oligodendroglial-like cells and fields of microcyst formation (Figure 6A), this glial component shows immunoreactivity for glial fibrillary acidic protein (Figure 5D). Rosenthal fibers and eosinophilic granular bodies are not observed, nor is the IDH-1 R132H mutation identified by immunohistochemistry. Mitotic figures are absent and proliferation analysis utilizing MIB-1 antibody discloses a labelling index of approximately $3 \%$ in Case 1 and less than $1 \%$ in Case 2. However, in both cases the small biopsied tissues were not enough for $1 \mathrm{p} / 19 \mathrm{q}$ testing after they were used for the routine studies. The oligodendrogliomalike genetic profile is unlikely to be seen in RGNT ${ }^{3}$, given the nature of this entity with those typical diagnostic features particularly of neurocytes forming rosettes and absence of IDH1 R132 mutation.

\section{Discussion}

The differential diagnosis of posterior third ventricular lesions can be challenging due to the diversity of tumors that arise or extend into this region, either from the third ventricular wall or the pineal region, velum interpositum, surrounding neural parenchyma, tentorium, or the posterior cerebral vasculature ${ }^{4}$. Typical lesions include: astrocytoma, ependymoma, epidermoid tumor, craniopharyngioma, meningiomas or metastasis ${ }^{4}$. Lesions that are extending from the pineal region are generally either germ cell tumors or pineal parenchymal tumors, although glial tumors and metastasis can also be included in the differential diagnosis for this location ${ }^{4}$.

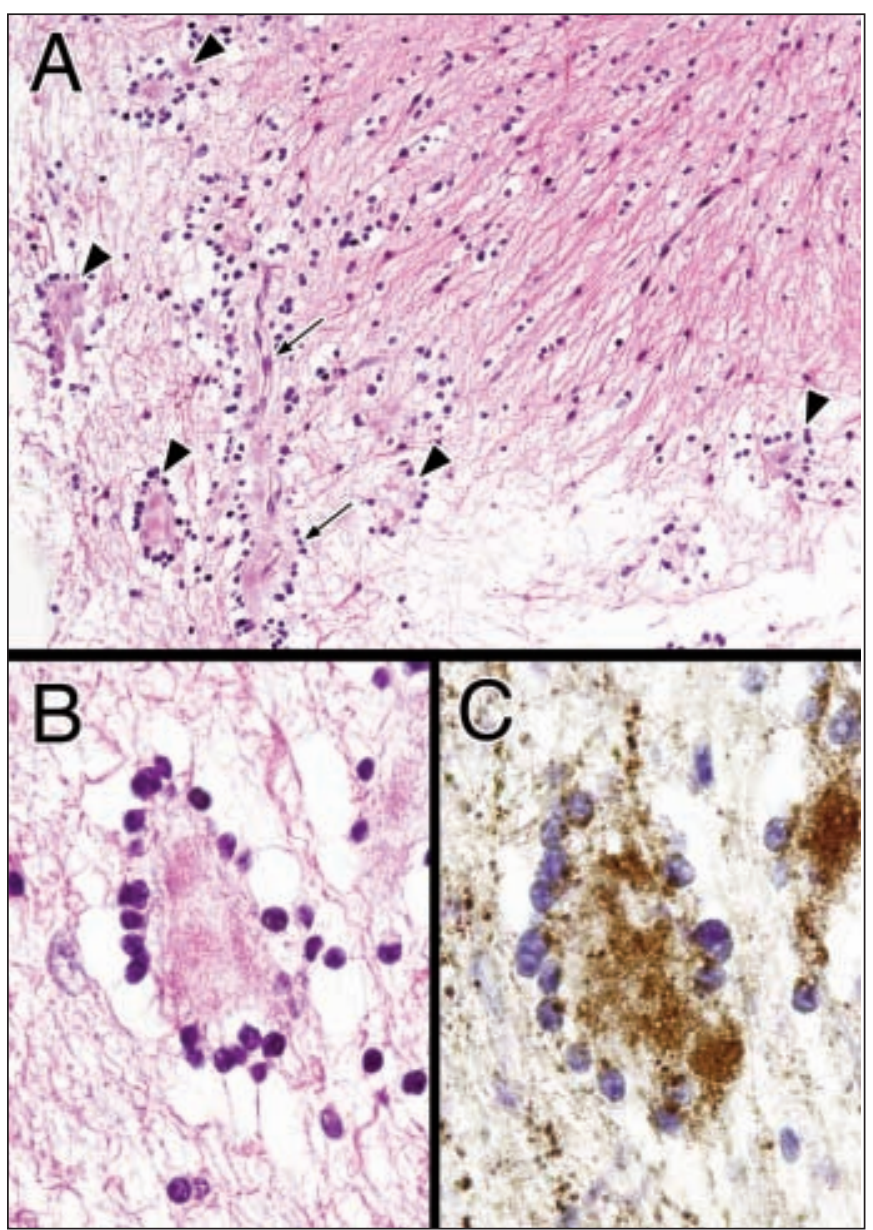

Figure 6: Findings of rosette forming glioneuronal tumor, Case 2. A. Low magnification of tumor showing in the upper right corner the piloid component of compacted spindled cells merging into the neurocytic component with microcyst formation (lower right corner) and neurocytic rosettes (arrowheads) with a perivascular pseudorosette (short arrows), on the left (H\&Ex 200). B. High magnification of a neurocytic rosette formed by a circle of neurocytes around a flocculent eosinophilic core of neuropil (H\&E x 1000). C. Marked immunoreactivity for synaptophysin is expressed by the neuropil core of the neurocytic rosette (Synaptophysin Immunohistochemistry $x$ 1000).

Rosette-forming glioneuronal tumor was included in the WHO Classification of the central nervous system tumors in $2007^{1}$. In 1995, prior to the WHO designation, Kuchelmeister et al described similar RGNT pathological findings as a form of dysembryoplastic neuroepithelial tumor ${ }^{1}$. In 2002, Komari et al reported 11 cases that had two pathological components. Their paper was the first to use the term rosette-forming glioneuronal tumor $^{1,5}$. Initial findings in 1998 described this lesion as a papillary neuroglial tumor. ${ }^{6}$ Since the inclusion of RGNT in the WHO Classification system, 49 cases have been reported in the English literature prior to April $2012^{2}$.

Rosette-forming glioneuronal tumor are characterized as rare slowly growing tumors ${ }^{1}$, occurring predominantly in the fourth ventricular region ${ }^{2}$, mostly affecting young adults and causing symptoms related to obstructive hydrocephalus ${ }^{1,2}$. This tumor 
tends to be in the midline with the most common location being the fourth ventricle and aqueduct ${ }^{1,2}$. Among 48 reported cases, 35 $(72 \%)$ were in the fourth ventricle or vermis ${ }^{2}$. The second most common locations are the pineal area and tectum ${ }^{1,2}$ and in the pineal gland with extension to the third and fourth ventricles ${ }^{7}$. Other posterior fossa locations such as the cerebellar hemispheres and the cerebellopontine angle are reported ${ }^{2}$. In the supratentorial region, these tumors are exceedingly rare with one case report of optic nerve RGNT in NF1 patient ${ }^{1}$, another in the septum pellucidum ${ }^{3}$, and a third patient with multiple lesions within the lateral ventricles.

This tumor was also reported in association with Noonan syndrome $^{8}$. Other genetic abnormalities with RGNT have been reported, including mutation of IDH 1 and IDH2 genes ${ }^{1}$, and specifically IDH $1 \mathrm{R} 132 \mathrm{H}^{3}$. Rosette-forming glioneuronal tumor does not possess distinguishing radiological features. It shares general benign tumor characteristics including isointense or hypointense pattern on T1 MRI sequence and hyperintense on T2 sequence with either ring enhancing or heterogeneous enhancement pattern on post-gadolinium T1 sequence ${ }^{1}$.

In terms of management, RGNT has been treated as a benign tumor where both gross total and subtotal resections have been reported $^{1}$. Tumor biopsy only and follow-up is advocated, especially in locations where major neurological deficit might results from resection ${ }^{1}$. The role of radiation therapy remains uncertain $^{1}$. There are only two cases of RGNT where radiation therapy is perused: one patient with disseminated intraventricular RGNT and another patient that received radiation therapy shortly after subtotal resection ${ }^{1}$.

\section{Conclusion}

Our paper is the first to report the occurrence of RGNT in the posterior third ventricle. RGNT should be considered as a diagnostic possibility for tumors outside the posterior fossa.

\section{REFERENCES}

1. Solis OE, Mehta RI, Lai A, et al. Rosette-forming glioneuronal tumor: a pineal region case with IDH1 and IDH2 mutation analyses and literature review of 43 cases. J Neurooncol. 2011; 102(3):477-84.

2. Hsu C, Kwan G, Lau Q, Bhuta S. Rosette-forming glioneuronal tumour: imaging features, histopathological correlation and a comprehensive review of literature. Br J Neurosurg. 2012;26(5): 668-73.

3. Xiong J, Liu Y, Chu SG, et al. Rosette-forming glioneuronal tumor of the septum pellucidum with extension to the supratentorial ventricles: rare case with genetic analysis. Neuropathology. 2012;32(3):301-5.

4. Lozier AP, Bruce JN. Surgical approaches to posterior third ventricular tumors. Neurosurg Clin N Am. 2003;14(4):527-45.

5. Komori T, Scheithauer BW, Hirose T. A rosette-forming glioneuronal tumor of the fourth ventricle: infratentorial form of dysembryoplastic neuroepithelial tumor? Am J Surg Pathol. 2002;26(5):582-91.

6. Komori T, Scheithauer BW, Anthony DC, et al. Papillary glioneuronal tumor: a new variant of mixed neuronal-glial neoplasm. Am J Surg Pathol.1998;22(10):1171-83.

7. Xu J, Yang Y, Liu Y, et al. Rosette-forming glioneuronal tumor in the pineal gland and the third ventricle: a case with radiological and clinical implications. Quant Imaging Med Surg. 2012;2(3): 227-31.

8. Karafin M, Jallo GI, Ayars M, Eberhart CG, Rodriguez FJ. Rosette forming glioneuronal tumor in association with Noonan syndrome: pathobiological implications. Clin Neuropathol. 2011;30(6):297-300. 